\title{
La escala de estilos de afrontamiento forma BMoos: validación en estudiantes universitarios mexicanos
}

\author{
Scale of Moos Type-B Coping Styles: Validation among \\ Mexican University Students
}

\author{
Jesús Javier Higareda Sánchez* \\ Arturo del Castillo Arreola* \\ Angélica Romero Palencia* \\ Flor de María Erari Gil Bernal* \\ Sofía Rivera Aragón** \\ *Universidad Autónoma del Estado de Hidalgo \\ * *Universidad nacional Autónoma de México
}

\section{RESUMEN}

En este estudio participaron 618 estudiantes de nuevo ingreso del Instituto de Ciencias de la Salud de la Universidad Autónoma del Estado de Hidalgo, pertenecientes a las carreras de psicología, medicina, odontología, nutrición, gerontología y farmacia. Fueron seleccionados mediante un muestreo no probabilístico por conveniencia. A través de la discriminación de ítems, el análisis factorial para obtener la validez de constructo y el análisis Alfa de Cronbach para la consistencia interna, se obtuvieron tres escalas válidas y confiables para universitarios mexicanos: la escala de estilos de afrontamiento personal $(\alpha=0.82)$; la escala de conductas negativas de afrontamiento $(\alpha=0.71)$ y la escala de Red de apoyo $(\alpha=0.81)$.

Palabras clave: afrontamiento, estudiantes, psicometría, escala, estrés

\section{ABSTRACT}

This study involved the participation of 618 newly enrolled students at the Autonomous University of the State of Hidalgo's Health Sciences Institute in the departments of psychology, medicine, dentistry, nutrition, gerontology and pharmaceutical sciences. They were selected through non-probabilistic convenience sampling. By discriminating items, the factor analysis to obtain the validity of the construct and the Cronbach's Alfa analysis to ensure internal consistency, three valid and reliable scales were obtained for the Mexican university students: the scale of personal coping styles $(\alpha=0.82)$; the scale of negative coping behaviors $(\alpha=0.71)$ and the support network scale $(\alpha=0.81)$.

Key words: coping, students, psychometry, scale, stress 
Para definir qué son los estilos de afrontamiento al estrés es necesario conocer cómo define la literatura el estrés psicológico; lo entiende como la relación particular entre la persona y el ambiente, el cual es evaluado por el individuo, como excedente de sus recursos, poniendo en riesgo su bienestar (Lazarus \& Folkman, 1984; Lazarus, 2006).

En el modelo que toma en cuenta el estrés como una evaluación cognitiva surge el concepto de estilos de afrontamiento al estrés, que son especificados como todos aquellos esfuerzos cognitivos y conductuales que la persona ocupa para manejar las demandas de un evento evaluado como estresante (Folkman, Lazarus, Pimley \& Novacek, 1987; Lazarus \& Folkman, 1984; Lazarus, 2006).

Los estilos de afrontamiento están clasificados como: afrontamiento centrado en el problema, donde los esfuerzos mentales y conductuales alteran directamente el ambiente para solucionar el evento estresante, y el afrontamiento centrado en la emoción, que regula las emociones surgidas al momento (Lazarus \& Folkman, 1984). Los autores de esta teoría mencionan que, ante cualquier situación estresante, las personas usarán algún tipo de estilo de afrontamiento (Folkman, Lazarus, Dunkel-Schetter, De Longis \& Gruen, 1986). Son Moos y Shaefer (1986) incluyen el estilo de afrontamiento centrado en la evaluación del problema, el cual busca modificar el significado de una situación que es evaluada como problemática, como el análisis lógico, la redefinición cognitiva y la evitación cognitiva.

La función del afrontamiento está asociada a la toma de decisiones, la búsqueda y la evaluación de información proveniente del ambiente para llevar a cabo acciones, mantener autonomía o libertad de movimiento y enfocar tanto los recursos como la energía hacia las demandas que presenta un evento estresante (Billings \& Moos, 1984; Janis \& Mann, 1977; Mechanic, 1974; White, 1974).

El afrontamiento ha sido evaluado por medio de entrevistas semiestructuradas como la usada por Billings, Folkman, Acree y Moskowitz (2000), quienes usaron como base la Escala de estilos de afrontamiento (Folkman \& Lazarus, 1988) que evalúa la reevaluación positiva, planeación de la solución del problema, soporte social, afrontamiento confrontativo, distanciamiento, evitación y escape cognitivo, evitación y escape conductual. Por tratarse de una entrevista no cuenta con datos de confiabilidad y validez.

Otra forma de evaluarlos ha sido por medio de los cuestionarios abiertos autoadministrados como el usado por Kasayira, Chipandambira y Hungwe (2007) en Zimbabwe; éste evalúa estresores y afrontamiento. Únicamente se usaron frecuencias para analizar los resultados obtenidos.

Por medio de inventarios como el cope Inventory (Carver, Scheier \& Weintraub, 1989), en su versión breve (Carver, 1997) emplea una escala Likert de cuatro puntos (no he estado haciendo esto en absoluto hasta he estado haciendo esto mucho). Los ítems fueron factorizados en: centrado en el problema como afrontamiento activo, planificación instrumental de apoyo, y religiosidad $(\alpha=0.80)$; afrontamiento activo emocional en forma de ventilación, reencuadre positivo, humor, aceptación, y apoyo emocional $(\alpha=0.81), y$ afrontamiento emocional por evitación en forma de autodistracción, negación, desconexión conductual, autoculpa, y uso de sustancias $(\alpha=0.88)$. Este inventario ha sido ocupado para evaluar estudiantes universitarios de Estados Unidos (Schnider, Elhai \& Gray, 2007).

Otra escala es la creada por Tobin, Holroyd y Reynolds (1989), que evalúa los estilos de afrontamiento por medio de 72 ítems tipo Likert; los factores son: solución de problemas, restructuración cognitiva, apoyo social, expresión emocional, evitación de problemas, pensamientos deseables, evitación social y autocrítica (con índices de confiabilidad entre ( $\alpha=.85$ y .98), la cual también ha sido usada en población universitaria de Estados Unidos (Penland, Masten, Zelhart, Fournet \& Callahan, 2000).

La Escala de estilos de afrontamiento de Moos ha sido adaptada para población adulta en Argentina (Mikulic \& Crespi, 2008), y mostró valores de confiabilidad y validez aceptables $(\alpha=.85)$. Por medio de un análisis de componentes principales (rotación Oblimin) fueron identificados ocho estilos de afrontamiento: resolución de problemas, análisis lógico, revalorización positiva, búsqueda de apoyo, búsqueda de gratificación, aceptación/resignación, evitación cognitiva y descarga emocional.

En México se ha usado la Escala de estilos de afrontamiento forma B de Moos, traducida y adaptada para adultos (Ayala \& Sánchez-Sosa, 1997 en Riveros, 
Cortázar-Palapa, Alcázar \& Sánchez-Sosa, 2005), la cual parte de la Escala de salud y vida cotidiana, forma B de Moos (Billings \& Moos, 1984). Se trata de una escala de 35 ítems distribuidos en ocho factores. Las primeras cuatro escalas se refieren a estilos basados en la aproximación al problema (análisis lógico, reevaluación positiva, búsqueda de guía y soporte y resolución de problemas), las cuatro restantes evalúan estilos de evitación o centrados en la emoción (evitación cognitiva, aceptación-resignación, búsqueda de recompensas alternativas y descarga emocional). No se reportan datos de validez y confiabilidad para población mexicana. Esta escala se ha empleado en varios estudios para población mexicana (Del Castillo, Martinez, García \& Guzmán, 2012; Del Castillo, Morales \& Solano, 2013), aunque no se han probado sus características psicométricas en dicha población.

De igual forma se han encontrado escalas para población adulta, como la creada por Góngora y Reyes (1998) para población yucateca, titulada Escala multidimensional de estilos de enfrentamiento, cuyos factores son: directo revalorativo, emocional negativo y evasivo, con un Alpha de Cronbach total de .74. Sin embargo, no reportan datos en población universitaria.

En la búsqueda bibliográfica no se encuentran instrumentos que evalúen los estilos de afrontamiento al estrés en población estudiantil universitaria mexicana que sean válidos y confiables. Por ello, el objetivo de este estudio fue validar una escala de estilos de afrontamiento al estrés, para ser aplicada en ambientes escolares universitarios.

\section{MÉTODO}

\section{Participantes}

Participaron 618 estudiantes de recién ingreso del Instituto de Ciencias de la Salud, de la Universidad Autónoma del Estado de Hidalgo, 435 mujeres que representan $70 \%$ de la muestra y 183 hombres que constituyen $30 \%$ de ella. De acuerdo con la distribución por licenciatura de los participantes, psicología contó con 111 alumnos, medicina 114, enfermería 99, gerontología 98, odontología, 96, nutrición 79 y farmacia 21. Los participantes fueron seleccionados mediante un muestreo no probabilístico por conveniencia. La media de edad fue $19.03(D . E .=1.43)$, con un rango de edad de 17 a 26 años.

\section{Instrumentos}

Se utilizó la Escala de estilos de afrontamiento personal de Moos traducida al castellano y adaptada para población mexicana por Ayala y Sánchez-Sosa (1997, en Riveros et al., 2005) tomada de la Escala de salud y vida cotidiana, forma B de Moos (Billings \& Moos, 1984). Evalúa ocho estilos de afrontamiento. Los primeros cuatro están centrados en la solución del problema (análisis lógico, reevaluación positiva, búsqueda de guía y soporte y resolución de problemas), y los cuatro restantes evalúan estilos centrados en la emoción (evitación cognitiva, aceptación-resignación, búsqueda de recompensas alternativas y descarga emocional). Se compone de 35 reactivos, con una escala de respuesta de seis opciones a elegir de "nunca" a "siempre" o "casi siempre".

\section{Procedimiento}

Se gestionó la aplicación en la Universidad Autónoma del Estado de Hidalgo con las áreas académicas de las licenciaturas de psicología, medicina, odontología, nutrición, gerontología y farmacia, como ya se mencionó. Las alumnas y los alumnos firmaron un consentimiento informado para aceptar participar en la investigación. Al finalizar la aplicación se realizó la captura, la revisión y la limpieza de la base de datos. Posteriormente, se realizó el análisis estadístico con el programa SPSS v. 21 para la obtención de resultados.

\section{RESULTADOS}

Para efectuar la validación de la escala de estilos de afrontamiento personal forma B de Moos para universitarios mexicanos se llevaron a cabo tres etapas o fases: la primera consistió en el análisis de discriminación de los reactivos, la segunda, en la obtención de la validez de constructo a través de un análisis factorial exploratorio, y la última, en la obtención de los coeficientes de consistencia interna.

\section{Discriminación de reactivos}

Puesto que todos los ítems obtuvieron respuestas en todas las opciones, se eliminaron aquellos reactivos que 
Tabla 1. Resultados del proceso de discriminación de los ítems

\begin{tabular}{|c|c|c|c|}
\hline Ítem & $\begin{array}{c}\text { Correlación con escala } \\
\text { total }\end{array}$ & Grupos extremos (t) & Distribución (Curtosis) \\
\hline $\begin{array}{l}\text { Trato de conocer más sobre el } \\
\text { problema }\end{array}$ & $.221 * *$ & .000 & -.321 \\
\hline $\begin{array}{l}\text { Hablo con mi pareja o mi familia } \\
\text { sobre el problema }\end{array}$ & $.161^{* *}$ & .000 & .163 \\
\hline $\begin{array}{l}\text { Hablo con un amigo acerca del } \\
\text { problema }\end{array}$ & $.165^{* *}$ & .000 & -.589 \\
\hline $\begin{array}{l}\text { Hablo con un profesional } \\
\text { (medico, abogado, etc.) del } \\
\text { problema }\end{array}$ & $.234^{* *}$ & .000 & -.878 \\
\hline $\begin{array}{l}\text { Le pido a Dios que me guíe o me } \\
\text { de valor }\end{array}$ & $.125^{* *}$ & .000 & -1.263 \\
\hline Me preparo por si pasa lo peor & $.211 * *$ & .000 & -.618 \\
\hline $\begin{array}{l}\text { No me preocupo, pienso que } \\
\text { todo saldrá bien }\end{array}$ & $.141 * *$ & .000 & -.879 \\
\hline $\begin{array}{l}\text { Cuando me siento enojado(a) me } \\
\text { desquito con otras personas }\end{array}$ & $.098^{*}$ & .003 & -.222 \\
\hline $\begin{array}{l}\text { Cuando me siento deprimido (a) } \\
\text { me desquito con otras personas }\end{array}$ & .071 & .008 & .705 \\
\hline $\begin{array}{l}\text { Trato de ver el lado bueno del } \\
\text { problema }\end{array}$ & $.188^{* *}$ & .000 & -.649 \\
\hline $\begin{array}{l}\text { Hago otras cosas para no pensar } \\
\text { en el problema }\end{array}$ & $.148^{* *}$ & .000 & -.505 \\
\hline $\begin{array}{l}\text { Pienso en un plan de acción y lo } \\
\text { llevo a cabo }\end{array}$ & $.184^{* *}$ & .000 & -.230 \\
\hline $\begin{array}{l}\text { Pienso en varias formas de } \\
\text { resolver el problema }\end{array}$ & $.214^{* *}$ & .000 & .305 \\
\hline $\begin{array}{l}\text { Me guío por mis experiencias } \\
\text { pasadas en situaciones similares }\end{array}$ & $.266^{* *}$ & .000 & .085 \\
\hline Doy a notar lo que siento & $.178^{* *}$ & .000 & -.905 \\
\hline $\begin{array}{l}\text { Trato de analizar el problema y } \\
\text { de ver las cosas objetivamente }\end{array}$ & $.242 * *$ & .000 & -.137 \\
\hline $\begin{array}{l}\text { Pienso en el problema, para tratar } \\
\text { de entenderlo mejor }\end{array}$ & $.153^{* *}$ & .000 & .327 \\
\hline $\begin{array}{l}\text { Trato de no precipitarme y no } \\
\text { actuar impulsivamente }\end{array}$ & $.153^{* *}$ & .000 & -.177 \\
\hline $\begin{array}{l}\text { Me digo cosas a mí mismo(a) } \\
\text { para sentirme mejor }\end{array}$ & $.121^{* *}$ & .000 & -.429 \\
\hline $\begin{array}{l}\text { Me olvido del problema por un } \\
\text { tiempo }\end{array}$ & 0.087 & .000 & -.800 \\
\hline
\end{tabular}




\begin{tabular}{|c|c|c|c|}
\hline Ítem & $\begin{array}{c}\text { Correlación con escala } \\
\text { total }\end{array}$ & Grupos extremos (t) & Distribución (Curtosis) \\
\hline $\begin{array}{l}\text { Sé lo que debo hacer, me } \\
\text { esfuerzo para que salgan bien las } \\
\text { cosas }\end{array}$ & $.163 * *$ & .000 & .705 \\
\hline $\begin{array}{l}\text { Prefiero estar alejado(a) de la } \\
\text { gente }\end{array}$ & .051 & .011 & -.698 \\
\hline $\begin{array}{l}\text { Me propongo que la próxima vez } \\
\text { las cosas serán diferentes }\end{array}$ & $.141 * *$ & .000 & .263 \\
\hline $\begin{array}{l}\text { Me niego a creer lo que está } \\
\text { pasando }\end{array}$ & .055 & .006 & -.265 \\
\hline $\begin{array}{l}\text { Si no puedo hacer nada para } \\
\text { cambiar las cosas, las acepto } \\
\text { como vienen }\end{array}$ & $.184 * *$ & .000 & -.587 \\
\hline $\begin{array}{l}\text { Trato de permanecer insensible, } \\
\text { que no me afecte }\end{array}$ & $.125^{* *}$ & .000 & -.741 \\
\hline $\begin{array}{l}\text { Busco ayuda con personas o } \\
\text { grupos que hubieran tenido } \\
\text { experiencias similares }\end{array}$ & $.174 * *$ & .000 & -1.104 \\
\hline $\begin{array}{l}\text { Trato de llegar a un acuerdo para } \\
\text { rescatar algo positivo }\end{array}$ & $.238 * *$ & .000 & -.301 \\
\hline $\begin{array}{l}\text { Trato de relajarme o tomarlo con } \\
\text { calma }\end{array}$ & $.191 * *$ & .000 & -.088 \\
\hline Bebiendo más & $.132 * *$ & .000 & 4.576 \\
\hline Comiendo más & $.103^{*}$ & .000 & 1.905 \\
\hline Fumando más & .007 & .049 & 5.068 \\
\hline Durmiendo más & $.094^{*}$ & .000 & -.337 \\
\hline Haciendo más ejercicio & $.128 * *$ & .000 & -.182 \\
\hline $\begin{array}{l}\text { ¿Cuántos amigos (as) consideras } \\
\text { que tienes? }\end{array}$ & $.116^{*}$ & .007 & .513 \\
\hline $\begin{array}{l}\text { ¿Cuántos amigos (as) cercanos } \\
\text { con quienes puedas platicar de } \\
\text { tus problemas tienes? }\end{array}$ & $.118^{* *}$ & .000 & -1.067 \\
\hline $\begin{array}{l}\text { ¿A cuántas personas conoces de } \\
\text { quienes puedas esperar ayuda } \\
\text { real cuando tienes problemas? }\end{array}$ & $.101^{*}$ & .000 & -1.074 \\
\hline $\begin{array}{l}\text { Tengo un círculo de amigos } \\
\text { (as) con quienes mantengo un } \\
\text { contacto cercano }\end{array}$ & -.036 & .914 & 3.748 \\
\hline
\end{tabular}

Nota: los resultados marcados en negritas son aquellos que no cubrieron el criterio correspondiente. 
no cumplieron con dos de tres criterios requeridos: Correlación de cada ítem con escala total.

Discriminación significativa entre el grupo extremo bajo y el grupo extremo alto del reactivo, obtenida mediante la prueba $t$ de student.

Curtosis del reactivo menor o igual a 1.0.

Del total de los 38 ítems de la escala se eliminaron el 32 y el 38 por no cumplir con dos de los tres criterios requeridos. De la misma forma, dado su tipo de respuesta y su contenido conceptual se decidió analizar por separado los ítems 30 al 37 en el análisis factorial.

Tabla 2. Resultados de la prueba de esfericidad y KMO

Kaiser-Meyer-Olkin

Aprox. Chi-Cuadrada

.872

\begin{tabular}{ccc}
\hline Test de esfericidad de Bartlett & Aprox. Chi-Cuadrada & 4296.992 \\
\hline Gl & 406 \\
\hline Sig. & .000 \\
\hline
\end{tabular}

Tabla 3. Estructura factorial de la Escala de estilos de afrontamiento al estrés

\begin{tabular}{l|c|c|c|c|c|c}
\multicolumn{1}{c|}{ Ítem } & $\mathbf{1}$ & $\mathbf{2}$ & $\mathbf{3}$ & $\mathbf{4}$ & $\mathbf{5}$ & $\mathbf{6}$ \\
\hline Trato de analizar el problema y de ver las cosas objetivamente & .80 & -.05 & .13 & .06 & .23 & .06 \\
\hline Pienso en un plan de acción y lo llevo a cabo & .80 & -.12 & -.01 & .14 & .04 & .19 \\
\hline Pienso en varias formas de resolver el problema & .78 & -.16 & .10 & .18 & .02 & .15 \\
\hline Pienso en el problema para tratar de entenderlo mejor & .76 & .00 & .22 & .08 & .11 & -.03 \\
\hline Me guío por mis experiencias pasadas en situaciones similares & .71 & -.01 & .07 & .11 & .13 & .07 \\
\hline Trato de no precipitarme y no actuar impulsivamente & .53 & -.12 & .23 & .08 & .28 & .05 \\
\hline Trato de llegar a un acuerdo para rescatar algo positivo & .44 & -.14 & .07 & .21 & .30 & .23 \\
\hline Cuando me siento deprimido(a) me desquito con otras personas & -.05 & .76 & .01 & .12 & -.15 & -.01 \\
\hline Cuando me siento enojado(a) me desquito con otras personas & -.14 & .75 & -.07 & .22 & -.12 & .00 \\
\hline Prefiero estar alejado(a) de la gente & -.04 & .61 & -.07 & -.21 & .23 & .15
\end{tabular}

60 I Psicología Iberoamericana I Jesús Higareda Sánchez · Arturo del Castillo Arreola · Angélica Romero Palencia · Flor de María Erari Gil Bernal · Sofía Rivera Aragón
Validez de constructo

Se aplicó un análisis factorial exploratorio de componentes principales para determinar la validez de constructo de la escala. La prueba de Kaiser-Meyer-Olkin (кмо) reveló que la matriz era factorizable $($ кмо $=$ .872) (vid. tabla 2). Los reactivos obtuvieron comunalidades por arriba de .40 , lo que indicó que miden un mismo constructo. Se eligieron seis factores con un valor propio mayor de 1 , los cuales explicaban $53.45 \%$ de la varianza, con un Alfa de Cronbach de .826 .

Se llevó a cabo una rotación ortogonal (Varimax) y se optó por aquellos reactivos que tenían un peso factorial mayor o igual a .40 para conformar el instrumento final. La versión consta de 28 reactivos (vid. tabla 3). 


\begin{tabular}{|c|c|c|c|c|c|c|}
\hline Ítem & 1 & 2 & 3 & 4 & 5 & 6 \\
\hline Me niego a creer lo que está pasando & -.18 & .55 & .12 & -.23 & .18 & .16 \\
\hline Hablo con un amigo acerca del problema & .07 & -.01 & .67 & .06 & .09 & .08 \\
\hline Hablo con mi pareja o mi familia sobre el problema & .31 & -.12 & .61 & .15 & -.12 & .10 \\
\hline Hablo con un profesional (medico, abogado, etc.) del problema & .14 & .05 & .55 & -.12 & .14 & -.13 \\
\hline Trato de conocer más del problema & .48 & -.06 & .50 & .12 & .16 & -.24 \\
\hline Doy a notar lo que siento & .38 & .26 & .42 & -.08 & -.16 & .23 \\
\hline Me propongo que la próxima vez las cosas serán diferentes & .23 & -.02 & -.04 & .72 & .13 & .07 \\
\hline Me preparo por si pasa lo peor & .14 & .11 & .14 & .61 & .26 & -.11 \\
\hline Sé lo que debo hacer, me esfuerzo para que salgan bien las cosas & .41 & -.18 & .11 & .55 & .18 & .15 \\
\hline Le pido a Dios que me guíe o me de valor & -.09 & .14 & .42 & .46 & -.05 & .03 \\
\hline Trato de permanecer insensible, que no me afecte & .21 & .28 & -.22 & .03 & .65 & -.09 \\
\hline No me preocupo, pienso que todo saldrá bien & .08 & -.13 & .29 & .14 & .57 & .26 \\
\hline $\begin{array}{l}\text { Si no puedo hacer nada para cambiar las cosas las acepto como } \\
\text { vienen }\end{array}$ & .19 & .03 & -.04 & .24 & .54 & .14 \\
\hline Trato de relajarme o tomarlo con calma & .35 & -.22 & .20 & .14 & .50 & .13 \\
\hline Hago otras cosas para no pensar en el problema & .13 & .04 & -.02 & .13 & .03 & .73 \\
\hline Me olvido del problema por un tiempo & .07 & .26 & .09 & -.11 & .19 & .72 \\
\hline Trato de ver el lado bueno del problema & .45 & -.29 & .06 & .05 & .26 & .48 \\
\hline \multirow[t]{2}{*}{ Me digo cosas a mí mismo(a) para sentirme mejor } & .29 & .04 & .34 & .35 & .13 & .37 \\
\hline & .872 & .653 & .638 & .615 & .600 & .641 \\
\hline$\%$ de varianza explicada & 24.4 & 8.7 & 6.2 & 5.3 & 4.6 & 4.1 \\
\hline$M$ & 3.44 & 1.49 & 3.04 & 3.25 & 2.90 & 2.92 \\
\hline D. E. & .931 & .954 & .865 & .942 & .946 & .935 \\
\hline
\end{tabular}

1. Afrontamiento cognitivo, 2. Afrontamiento baja tolerancia a la frustración y retirada social, 3. Afrontamiento externo, 4. Preparación para la acción, 5. Evitación del malestar, 6. Distracción.

Nota. El ítem 27 no cargó en ningún factor. Los pesos factoriales por ítem se encuentran marcados en negritas, dependiendo el factor al que correspondieran. 
Para las conductas negativas de afrontamiento, se aplicó un análisis factorial confirmatorio. La prueba de Kaiser-Meyer-Olkin reveló que la matriz era factorizable $($ кмо $=.726)($ vid. tabla 4$)$. Los reactivos obtuvieron comunalidades por arriba de .45 lo que indicó que miden un mismo constructo. Surgió únicamente un factor con un valor propio mayor de 1, que explicaba $46.15 \%$ de la varianza, con un índice AGFI de 0.97 y un índice RMSR de 0.06; esto indicó que se trata de una escala válida. Los cinco reactivos tuvieron un peso factorial mayor o igual a .46 (vid. tabla 5).

Finalmente, en cuanto a la red de apoyo (amigos con que cuentan los participantes) se llevó a cabo el mismo procedimiento. Se aplicó un análisis factorial confirmatorio. La prueba de Kaiser-Meyer-Olkin reveló que la matriz era marginalmente factorizable (кмо $=$.615) (vid. tabla 6). Los reactivos obtuvieron comunalidades por arriba de .49 , lo que indicó que miden un mismo constructo. Surgió únicamente un factor con un valor propio mayor de 1 , que explicaba $60.82 \%$ de la varianza, con un índice AGFI de 1.00 y un índice RMSR de 0.00 lo que índico una escala válida; los tres reactivos tuvieron un peso factorial mayor o igual a .49 (vid. tabla 7).

Así, la Escala de estilos de afrontamiento personal al estrés forma $\mathrm{B}$ de Moos es un instrumento que evalúa seis estilos de afrontamiento (26 reactivos), cinco conductas negativas de afrontamiento y la red de apoyo social de las personas ante situaciones de estrés (tres ítems).

\section{Consistencia interna}

Se realizó la prueba del Alpha de Cronbach con el fin de conocer la consistencia interna de las escalas ( $v i d$. tabla 8 ).

Tabla 4. Resultados de la prueba de esfericidad y KMO

\begin{tabular}{c|c|c} 
KMO y Test de Bartlett & & .725 \\
\hline Kaiser-Meyer-Olkin & & 417.0 \\
\hline Test de esfericidad de Bartlett & aprox. Chi-Cuadrada & 10 \\
\hline & Gl & .000 \\
\hline
\end{tabular}

Tabla 5. Estructura factorial de las conductas negativas de afrontamiento

\begin{tabular}{l|l} 
& 1 \\
\hline 31 Comiendo más & .64 \\
\hline 30 Bebiendo más & .63 \\
\hline 33 Durmiendo más & .56 \\
\hline 32 Fumando más & .55 \\
\hline 34 Haciendo más ejercicio & .46 \\
\hline
\end{tabular}

Tabla 6. Resultados de la prueba de esfericidad y KMO

\begin{tabular}{c|c|c} 
KMO y Test de Bartlett & & \\
\hline Kaiser-Meyer-Olkin & & .615 \\
\hline Test de esfericidad de Bartlett & aprox. Chi-Cuadrada & 247.3 \\
\hline & Gl & 3 \\
\hline & Sig. & .000 \\
\hline
\end{tabular}


Tabla 7. Estructura factorial de la red de apoyo

\begin{tabular}{l|c} 
& 1 \\
\hline $\begin{array}{l}36 \text { ¿Cuántos amigos(as) cercanos con quienes puedas } \\
\text { platicar de tus problemas tienes? }\end{array}$ & .88 \\
\hline $\begin{array}{l}37 \text { ¿Cuántas personas conoces de quienes puedas } \\
\text { esperar ayuda real cuando tienes problemas? }\end{array}$ & .57 \\
\hline 35 ¿Cuántos amigos(as) consideras que tienes? & .49 \\
\hline
\end{tabular}

Factor 1. Red de apoyo

Tabla 8 Coeficientes de consistencia interna de los factores

\begin{tabular}{l|c|c} 
& Alfa de Cronbach & Núm. de ítems \\
\hline Estilo de afrontamiento al estrés & & 7 \\
\hline Afrontamiento cognitivo & 0.872 & 4 \\
\hline $\begin{array}{l}\text { Baja tolerancia a la frustración y } \\
\text { retirada social }\end{array}$ & 0.653 & 5 \\
\hline Afrontamiento externo & 0.638 & 4 \\
\hline Preparación para la acción & 0.615 & 4 \\
\hline Evitación del malestar & 0.600 & 4 \\
\hline Distracción & 0.641 & 28 \\
\hline Total & 0.826 & 2 \\
\hline
\end{tabular}

\begin{tabular}{l|c|c}
\hline $\begin{array}{l}\text { Conductas negativas de } \\
\text { afrontamiento }\end{array}$ & \\
\hline Afrontamiento conductual & 0.718 & 5 \\
\hline
\end{tabular}

\begin{tabular}{l|c|c}
\hline Red de apoyo & & \\
\hline Apoyo social & 0.817 & 3 \\
\hline
\end{tabular}

\section{DISCUSIÓN}

Tal como lo indican Reyes-Lagunes y García y Barragán (2008) se llevó a cabo la validación psicométrica a través de los siguientes análisis: análisis de frecuencias, discriminación de reactivos para grupos extremos, confiabilidad interna y validez de constructo; con esto se logró tener una validación psicométrica culturalmente relevante para una escala de estilos de afrontamiento al estrés en universitarios mexicanos.

De acuerdo con los resultados observados, se obtuvo un instrumento válido y confiable que consta de tres subescalas: la escala de estilo de afrontamiento personal, la escala de conductas negativas y la escala de red de apoyo.

De acuerdo con la revisión bibliográfica, se observa que la escala de afrontamiento personal forma B de Moos, en su versión para estudiantes universitarios mexicanos, cuenta con características similares a otras escalas empleadas fuera de México (Carver, 1997; Folkman \& Lazarus, 1988; Mikulic \& Crespi, 2008; Tobin et al., 1989), como la división en: estilo de afrontamiento focalizado en el problema (afrontamiento cognitivo, afrontamiento externo y preparación para la acción) y focalizado en la emoción (baja tolerancia a 
la frustración y retirada social, evitación del malestar y distracción). Por el contrario, se identifica que la principal diferencia del instrumento consistió en la agrupación de los reactivos en tres escalas diferentes. Esta separación plantea la posibilidad de una aplicación agrupada o por separado de cada una de las escalas en futuros estudios, dependiendo de los objetivos, ya que permite conocer tres componentes teóricamente relevantes del afrontamiento: los estilos, las conductas negativas y la percepción de apoyo social, al momento de encontrarse en situaciones evaluadas como estresantes.

En esta muestra se observa que el estilo más usado por las alumnas y los alumnos es el afrontamiento cognitivo $(\overline{\mathrm{x}}=3.44)$ y por otro lado el afrontamiento baja tolerancia a la frustración con retirada social $(\bar{x}=$ 1.49), como el estilo menos usado. Los estilos de afrontamiento encontrados por arriba de la media teórica

\section{REFERENCIAS}

Billings, A.G. \& Moos, R.H. (1984). Coping, stress, and social resources among adults with unipolar depression. Journal of Personality and Social Psychology, 46(4), 877.

Billings, D.W., Folkman, S., Acree, M. \& Moskowitz, J.T. (2000). Coping and Physical Health During Caregiving: The Roles of Positive and Negative Affect. Journal of Personality and Social Psychology, 79(1), 131-142.

Carver, C.S. (1997). You want to measure coping but your protocol'too long: Consider the brief cope. International Journal of Behavioral Medicine, 4(1), 92-100.

Carver, C.S., Scheier, M.F. \& Weintraub, J.K. (1989). Assessing coping strategies: a theoretically based approach. Journal of Personality and Social Psychology, 56(2), 267.

Del Castillo, A., Martínez, C., García, M. \& Guzmán, R. (2012). Intervención cognitivo conductual para modificar el nivel de distress en pacientes con diabetes tipo 2. En: S. Galán \& E. Camacho (Eds.). Estrés y salud: investigación básica y aplicada. México: Manual Moderno.

Del Castillo, A., Morales, O. \& Solano, G. (2013). Malestar emocional y estrategias de afrontamiento en pacien- fueron: afrontamiento cognitivo $(\overline{\mathrm{x}}=3.44)$, afrontamiento externo $(\overline{\mathrm{x}}=3.04)$ y preparación para la acción $(\overline{\mathrm{x}}=3.25)$ y tres por debajo: evitación del malestar $(\overline{\mathrm{x}}=$ $2.90)$, distracción $(\overline{\mathrm{x}}=2.92)$ y afrontamiento baja tolerancia a la frustración y retirada social $(\overline{\mathrm{x}}=1.49)$. Con ello se denota que los estilos más usados en esta población son los focalizados en la solución de la problemática.

A partir de estos resultados surgen nuevas interrogantes a clarificar en próximos estudios tales como el efecto de la escolaridad, la deseabilidad social y el sexo en los estilos de afrontamiento y la relación de éstos con múltiples variables tales como: estilos parentales, habilidades sociales, autoeficacia, autocuidado y demás variables sociales y de salud. Ahora que se cuenta con una escala válida y confiable para la población universitaria mexicana será más viable el desarrollo de estas líneas de investigación. •

tes con diabetes tipo 2: evaluación e intervención. Revista Latinoamericana de Medicina Conductual/ Latin American Journal of Behavioral Medicine, 3(1), 24-30.

Folkman, S., \& Lazarus, R. (1988). The ways of coping questionnaire. Palo Alto, CA: Consulting Psychologists Press.

Folkman, S., Lazarus, R.S., Dunkel-Schetter, C., DeLongis, A. \& Gruen, R.J. (1986). Dynamics of a stressful encounter: cognitive appraisal, coping, and encounter outcomes. Journal of Personality and Social Psychology, 50(5), 992.

Folkman, S., Lazarus, R.S, Pimley, S. \& Novacek, J. (1987) Age differences in stres and coping processes. Psychology and Aging, 2(2), 171-184.

Góngora, E., \& Reyes, I. (1998). El enfrentamiento a los problemas y el locus de control. La Psicología Social en México, 8, 165-172.

Janis, I.L. \& Mann, L. (1977). Decision making: A psychological analysis of conflict, choice, and commitment. New York: Free Press.

Kasayira, J.M., Chipandambira, K.S., \& Hungwe, C. (2007). Stressors faced by university students and their coping strategies: A case study of midlands state university students in Zimbabwe. In Frontiers In Education Conference-Global Engineering: Knowledge 
Without Borders, Opportunities Without Passports, 2007. FIE'07. 37th Annual (pp. F1A-22). IEEE.

Lazarus, R. S. \& Folkman, S. (1984). Stress, appraisal and coping. New York: Springer.

Lazarus, R.S. (2006). Stress and emotion. New York: Springer.

Mechanic, D. (1974). Social structure and personal adaptation: Some neglected dimensions. Coping and adaptation, 32, 44 .

Mikulic, I.M., \& Crespi, M.C. (2008). Adaptación y validación del Inventario de Respuestas de Afrontamiento de Moos (CRI-A) para adultos. Anuario de investigaciones, 15. Recuperado de: http://www.scielo.org.ar/scielo.php?script=sci_artte xt\&pid=S1851-16862008000100030

Moos, R.H. \& Schaefer, J. A. (1986). Life transitions and crises: a conceptual overview. En: R.H. Moos (Ed.). Coping with life crises: an integrated approach. New York: Premium Press.

Penland, E.A., Masten, W.G., Zelhart, P., Fournet, G.P. \& Callahan, T.A. (2000). Possible Selves, Depression and Coping Skills in University Students. Personality and Individual Differences, 29(5), 963-969.
Reyes-Lagunes, I., \& García y Barragán, F. (2008). Hacia un procedimiento de validación psicométrica culturalmente relevante. La Psicología Social en México, 12, 625-630.

Riveros, A., Cortazar-Palapa, J., Alcazar, F. \& SánchezSosa, J.J. (2005). Efectos de una intervención cognitivo-conductual en la calidad de vida, ansiedad, depresión y condición médica de pacientes diabéticos e hipertensos esenciales. International Journal of Clinical and Health Psychology, 5(3), 445-462.

Tobin, D.L., Holroyd, K.A., Reynolds, R.V., \& Wigal, J.K. (1989). The hierarchical factor structure of the coping strategies inventory. Cognitive Therapy and Research, 13(4), 343-361.

Schnider, K.R., Elhai, J.D., \& Gray, M.J. (2007). Coping style use predicts posttraumatic stress and complicated grief symptom severity among college students reporting a traumatic loss. Journal of Counseling Psychology, 54(3), 344-350.

White, R.W. (1974). Strategies of adaptation: An attempt at systematic description. Coping and adaptation, 47, 68. 